\title{
Targeting of Monomer/Misfolded SOD1 as a Therapeutic Strategy for Amyotrophic Lateral Sclerosis
}

\author{
Hsueh-Ning Liu, ${ }^{1}$ Sonja Tjostheim, ${ }^{1}$ Kevin DaSilva, ${ }^{1}$ David Taylor, ${ }^{1}$ Beibei Zhao, ${ }^{1}$ Rishi Rakhit, ${ }^{3}$ Mary Brown, ${ }^{2}$ \\ Avijit Chakrabartty, ${ }^{3}$ JoAnne McLaurin, ${ }^{2}$ and Janice Robertson ${ }^{1,2}$ \\ ${ }^{1}$ Tanz Centre for Research in Neurodegenerative Diseases and ${ }^{2}$ Department of Laboratory Medicine and Pathobiology, University of Toronto, Toronto, \\ Ontario M5S 3H2, Canada, and ${ }^{3}$ Department of Biochemistry and Medical Biophysics, Ontario Cancer Institute, University of Toronto, Toronto, Ontario \\ M5G 1L7, Canada
}

There is increasing evidence that toxicity of mutant superoxide dismutase-1 (SOD1) in amyotrophic lateral sclerosis (ALS) is linked to its propensity to misfold and to aggregate. Immunotargeting of differently folded states of SOD1 has provided therapeutic benefit in mutant SOD1 transgenic mice. The specific region(s) of the SOD1 protein to which these immunization approaches target are, however, unknown. In contrast, we have previously shown, using a specific antibody [SOD1 exposed dimer interface (SEDI) antibody], that the dimer interface of SOD1 is abnormally exposed both in mutant SOD1 transgenic mice and in familial ALS cases associated with mutations in the SOD1 gene (fALS1). Here, we show the beneficial effects of an active immunization strategy using the SEDI antigenic peptide displayed on a branched peptide dendrimer to target monomer/misfolded in SOD1 ${ }^{\mathrm{G} 37 \mathrm{R}}$ and SOD1 ${ }^{\mathrm{G} 93 \mathrm{~A}}$ mutant SOD1 transgenic mice. Immunization delayed disease onset and extended disease duration, with survival times increased by an average of $40 \mathrm{~d}$ in SOD1 ${ }^{\mathrm{G} 37 \mathrm{R}}$ mice. Importantly, this immunization strategy favored a Th2 immune response, thereby precluding deleterious neuroinflammatory effects. Furthermore, the beneficial effects of immunization correlated with a reduction in accumulation of both monomer/misfolded and oligomeric SOD1 species in the spinal cord, the intended targets of the immunization strategy. Our results support that SOD1 misfolding/aggregation plays a central role in SOD1-linked ALS pathogenesis and identifies monomeric/misfolded SOD1 as a therapeutic target for SOD1-related ALS.

\section{Introduction}

Amyotrophic lateral sclerosis (ALS) is an adult-onset neurodegenerative disease characterized by the loss of motor neurons from the brain and spinal cord. Approximately $1-2 \%$ of ALS cases are caused by mutations in the gene encoding superoxide dismutase-1 (SOD1) (Rosen et al., 1993), in which >130 different mutations have now been identified (http://alsod.iop.kcl. ac.uk). There is increasing evidence that the toxicity of mutant SOD1 is linked to its propensity to misfold and to aggregate (Bruijn et al., 2004). Indeed, aggregated SOD1 is a detectable pathological feature in familial ALS cases caused by mutations in SOD1 (fALS1) and in mutant SOD1 transgenic mice (Bruijn et al., 2004). Recent reports also suggest that misfolded/aggregated SOD1 may be relevant to cases of sporadic ALS (Ezzi et al., 2007; Gruzman et al., 2007; Bosco et al., 2010). In earlier studies, we

\footnotetext{
Received 0ct. 5, 2011; revised April 17, 2012; accepted April 26, 2012.

Author contributions: H.-N.L., K.D., J.M., and J.R. designed research; H.-N.L., S.T., K.D., D.T., B.Z., R.R., and M.B. performed research; R.R. and A.C. contributed unpublished reagents/analytic tools; H.-N.L., S.T., K.D., A.C., J.M., and J.R. analyzed data; H.-N.L., A.C., J.M., and J.R. wrote the paper.

This work was supported by American ALS Association Grant ALSA1302 (J.M., J.R.), Canadian Institutes of Health Research (CIHR) Grant PRG:81125 (J.M.), CIHR (H.-N.L.), Natural Sciences and Engineering Research Council of Canada (K.D.), and The James Hunter and Family ALS Initiative. J.R. holds a Tier 2 Canada Research Chair in ALS. We thank Philip Wong (The Johns Hopkins University, Baltimore, MD) for the G37R transgenic mice.

The authors declare no competing financial interests.

Correspondence should be addressed to Dr. Janice Robertson, Centre for Research in Neurodegenerative Diseases, University of Toronto, 6 Queen's Park Crescent West, Toronto, Ontario M5S 3H2, Canada. E-mail: jan.robertson@utoronto.ca.

DOI:10.1523/JNEUROSCI.5053-11.2012

Copyright $\odot 2012$ the authors $\quad 0270-6474 / 12 / 328791-09 \$ 15.00 / 0$
}

described SOD1 exposed dimer interface (SEDI) antibody, which was raised to an epitope within a hydrophobic region normally buried in the native homodimeric structure of SOD1, but is exposed when the dimer is disrupted or becomes monomeric (Rakhit et al., 2007). As such, SEDI antibody selectively recognizes monomer/misfolded conformations, but not the native structure. Using SEDI antibody, we confirmed the presence of mono$\mathrm{mer} /$ misfolded SOD1 as a common pathological entity in mutant SOD1 transgenic mice (G37R, G93A, G85R) and in fALS1 (A4V, A4T, I113T, G27/P28, V14M) (Rakhit et al., 2007; Liu et al., 2009).

Both active and passive immunotargeting of variously folded states of SOD1 has been shown to have therapeutic benefits in mutant SOD1 transgenic mice (Urushitani et al., 2007; Gros-Louis et al., 2010; Takeuchi et al., 2010). For active immunization, recombinant apo-G85R or apo-WT was used as immunogen, based on prior evidence that this metal-free protein had an increased propensity to misfold (Urushitani et al., 2007; Takeuchi et al., 2010). However, this immunogen has epitopes representative of both natively folded and various nondefined misfolded forms of SOD1 (Urushitani et al., 2007; Takeuchi et al., 2010) and as such would likely target both native and mutant forms of the protein. For passive immunization, different monoclonal antibodies raised to recombinant G93A and recognizing mutant/misfolded forms of SOD1 were used (Gros-Louis et al., 2010). Although C4F6 and D3H5 are very interesting antibodies for the study of mutant/misfolded SOD1, the epitopes that they detect currently remain unknown, and as such it is unclear ex- 
actly what SOD1 species are being targeted. In contrast, the epitope of SEDI antibody is precisely known, amino acid residues 145-151, within the dimer interface of the natively folded SOD1 protein (Rakhit et al., 2007). We have shown using SEDI antibody that exposure of the dimer interface is common to all SOD1 mutants tested to date, including A4T, A4V, V14M, $\Delta \mathrm{G} 27 / \mathrm{P} 28$, G93A, G37R, G85R, and I113T (Rakhit et al., 2007; Liu et al., 2009), and therefore targeting of this interface may have broad applicability to ALS cases associated with mutations in SOD1. Here, we describe the beneficial effects of using SEDI peptide in an active immunization strategy to specifically target monomer/ misfolded SOD1 in mutant SOD1 transgenic mice.

\section{Materials and Methods}

Animal model. Two lines of transgenic mice overexpressing mutant human SOD1 were used in this study. SOD1 ${ }^{\mathrm{G} 37 \mathrm{R}}$ mice carrying the human SOD1 gene with a G37R mutation (line 29) were on a pure C57BL/6 background (Wong et al., 1995). SOD $1^{\text {G93A }}$ mice (B6.Cg-Tg[SOD1G93A]1Gur/J) purchased from The Jackson Laboratory express a high copy number of the human SOD1 containing the G93A substitution. Both mouse lines were maintained as hemizygotes by breeding transgenic males with C57BL/6 females. Transgenic progeny were identified by PCR using primers specific for human SOD1. All protocols were conducted in accordance with the Canadian Council on Animal Care.

Immunization protocol. SOD $1^{\mathrm{G} 37 \mathrm{R}}$ or SOD $1^{\mathrm{G} 93 \mathrm{~A}}$ transgenic mice were immunized intraperitoneally starting at 6 weeks of age. The immunogen used for the study was SEDI peptide designed as a multiple antigenic peptide, with each branch chain consisting of the sequence at the SOD1 dimer interface (residues 143-151) (Rakhit et al., 2007). One hundred micrograms of SEDI peptide antigen was emulsified 1:1 (v/v) with complete Freund's adjuvant (CFA) for the first immunization, followed by a boost in incomplete Freund's adjuvant at 2 weeks, and once monthly for the next 3 months for SOD $1^{\text {G37R }}$ mice. Two boosts were made after the initial prime for SOD1 ${ }^{\mathrm{G} 93 \mathrm{~A}}$ mice. Transgenic mice received PBS formulated in CFA followed by incomplete Freund's adjuvant as above for negative controls.

Antibody titers and isotyping. The antibody titer was assayed by ELISA in serum samples collected at 6 months of age and the end stage of disease. Microplates were coated with unfolded SOD1 to determine the antibody titer for mice immunized with SEDI peptide. SOD1 was unfolded overnight at room temperature in Tris-buffered saline, $\mathrm{pH}$ 7.4, containing $8 \mathrm{~m}$ urea, 2 mM DTT, and 1 mM EDTA (Rakhit et al., 2007). Serially diluted serum was allowed to bind overnight at $4^{\circ} \mathrm{C}$, followed by incubation of HRP-conjugated goat anti-mouse IgG (Pierce). The titer, defined as the dilution yielding $50 \%$ of the maximal signal, was detected spectrophotometrically at $405 \mathrm{~nm}$ after adding tetramethyl benzene substrate (Pierce). To determine antibody isotype, anti-mouse Ig subclassspecific HRP-conjugated secondary antibodies were added (Zymed Laboratories).

Tissue processing and axon counting. Anesthetized mice were flushperfused transcardially with PBS after CSF was collected. The lumbar segment of spinal cord was removed and processed for paraffin embedding or cryostat sectioning. L5 ventral roots were dissected out and postfixed in formalin. After incubating in 1\% osmium tetroxide, roots were dehydrated in graded series of ethanol and embedded in Epon (Marivac). Sections of $1 \mu \mathrm{m}$ were stained with toluidine blue and examined under a light microscope. Motor axons in the cross section of each L5 ventral root were counted, and diameters were determined with stereological microscopy.

Immunohistochemistry and immunofluorescence. Formalin-fixed, paraffin-embedded lumbar spinal cords were cut into $6 \mu \mathrm{m}$ transverse sections. Sections were labeled with hematoxylin and eosin (H\&E) or a rabbit polyclonal antibody against glial fibrillary acidic protein (GFAP) (1:1000; Dako). For immunohistochemical detection of activated microglia, formalin-fixed spinal cords were cryoprotected in 30\% sucrose in PBS. Ten micrometer cryosections were incubated with a rat monoclonal antibody against Mac-2 (1:100; Cedarlane) followed by biotinylated goat anti-rat IgG (1:200; Vector Laboratories) and biotin-avidin complex conjugated to HRP (1:100; Dako), and visualized with a DAB substrate kit (Vector Laboratories). For double immunofluorescent labeling, freshly dissected lumbar spinal cord was embedded in Tissue-Tek (OCT compound) and frozen in isopentane. Cryosections were incubated with the following antibodies: serum from vaccinated mice (1:100) and GFAP (1:2000; Dako). This was followed by incubation with species-specific secondary antibodies conjugated with Alexa Fluor dye (1:250; Invitrogen). Immunostaining was then visualized by fluorescence microscopy.

Western blotting. For detection of SOD1 oligomers, lumbar spinal cords from the vaccinated mice were prepared in nonreducing conditions as previously described (Furukawa et al., 2006). Ten micrograms of protein were separated on $12 \%$ SDS-PAGE. Following transfer onto PVDF membrane (Millipore), the blot was probed with an antibody to human SOD1 (S100; 1:1000; Nventa Biopharmaceuticals) followed by secondary goat anti-rabbit HRP-conjugated IgG (1:5000; GE Healthcare). Immunoreactivities were developed using ECL reagents (PerkinElmer) and quantified using NIH ImageJ software. The blots were reprobed with a monoclonal anti-GAPDH antibody (1:5000; Biodesign) to show equal loading. To determine whether sera generated from the immunized mice recognized human SOD1 in transgenic mice, spinal cords from nontransgenic (non-Tg) and SOD1 ${ }^{\text {G37R }}$ mice were homogenized in RIPA buffer $(50 \mathrm{~mm}$ Tris, pH 7.5, $150 \mathrm{~mm} \mathrm{NaCl}, 1 \mathrm{~mm}$ EDTA, $1 \% \mathrm{NP}-40$, and $0.25 \% \mathrm{Na}-$ deoxycholate). After centrifuging at $15,000 \times g$ for $10 \mathrm{~min}$, supernatants were electrophoresed on 12\% SDS-polyacrylamide gels and transferred to PVDF membranes. The membranes were incubated with sera $(1: 1000)$ from adjuvant- or SEDI-immunized mice followed by HRP-conjugated secondary antibodies (1:5000) and developed using chemiluminescence. An identical blot was probed with commercial antibodies against human SOD1 (S100; Nventa Biopharmaceuticals) and GAPDH.

Immunoprecipitation. To quantify the level of monomer/misfolded SOD1 in spinal cord of the vaccinated mice, protein extracts were incubated with SEDI antibody and $20 \mu \mathrm{l}$ of $50 \%(\mathrm{w} / \mathrm{v})$ protein A-agarose (Sigma-Aldrich) in RIPA buffer. Sera obtained from mice immunized with SEDI peptide were used to immunoprecipitate misfolded SOD1 from spinal cord extracts of non-Tg and SOD $1{ }^{\mathrm{G} 37 \mathrm{R}}$ mice. Immunoprecipitates were liberated by boiling in Laemmli sample buffer and analyzed by Western blotting with a polyclonal antibody to SOD1 (1:1000; Calbiochem). A separate blot loaded with $0.3 \%$ supernatant input was probed with antibodies to SOD1 (1:1000; Calbiochem) and GAPDH as loading controls.

Behavioral testing. The motor function of transgenic animals was assessed weekly using a rotarod task on an Economex Rotarod instrument (Columbus Instruments). Three trials were performed for each mouse, and the longest time taken to fall was recorded (Azzouz et al., 2004). Footprint analysis was performed by painting the hindpaws with ink and recording walking patterns (Azzouz et al., 2004). An average of four stride lengths were taken for each animal at the time point evaluated.

Statistical analysis. Statistical significance was performed using oneway ANOVA followed by Bonferroni's post hoc test. Kaplan-Meier survival analysis and log rank test were used for disease onset and survival comparisons. Repeated-measures ANOVA were used for rotarod comparisons. A value of $p<0.05$ was considered significant.

\section{Results}

\section{Immunization against monomer/misfolded SOD1 improves motor performance, delays disease onset, and extends survival in SOD $1^{\mathrm{G} 37 \mathrm{R}}$ mice}

To determine whether SOD1 was released into the extracellular space, we tested for the presence of SOD1 in the CSF from mutant SOD1 ${ }^{\text {G37R }}$ transgenic mice and non-Tg littermates by Western blot analysis. In agreement with findings in transgenic SOD 1 G93A rats (Turner et al., 2005), both human and endogenous mouse SOD1 was detected in the CSF of SOD ${ }^{\mathrm{G} 37 \mathrm{R}}$ mice, and mouse SOD1 in the non-Tg animals (Fig. $1 A$ ). More importantly, to test for the presence of monomer/misfolded SOD1 in the CSF, immunoprecipitation reactions were performed using the SEDI antibody. Monomeric/misfolded SOD1 was detected in the CSF of SOD $1^{\mathrm{G} 37 \mathrm{R}}$ mice and also of SOD $1^{\mathrm{G} 93 \mathrm{~A}}$ mice but not in the 
A

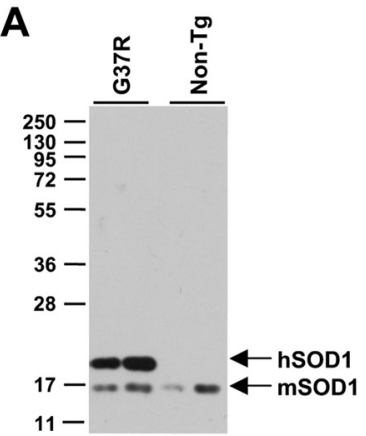

B
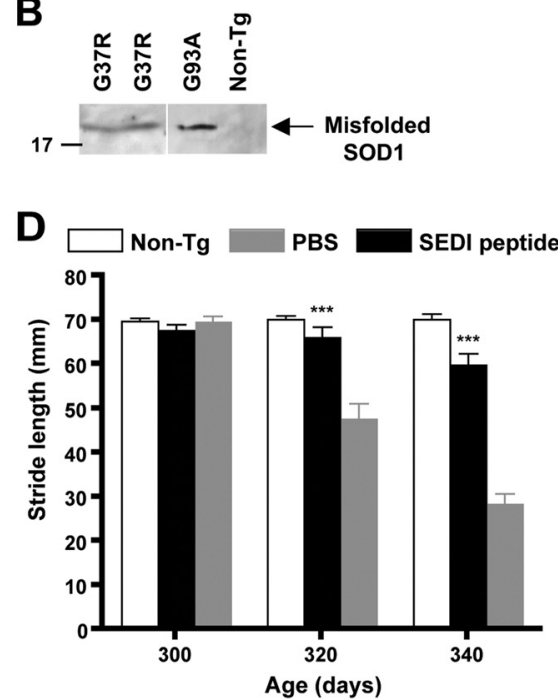

\section{E}
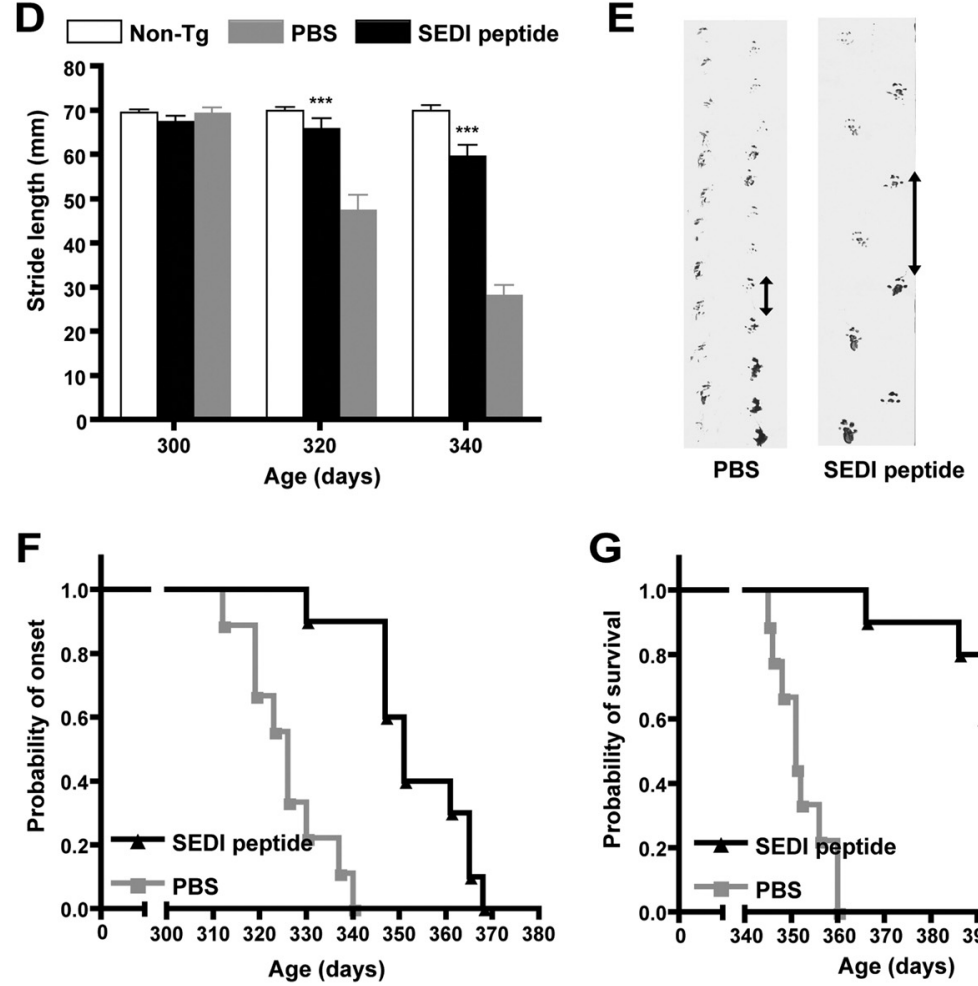

C

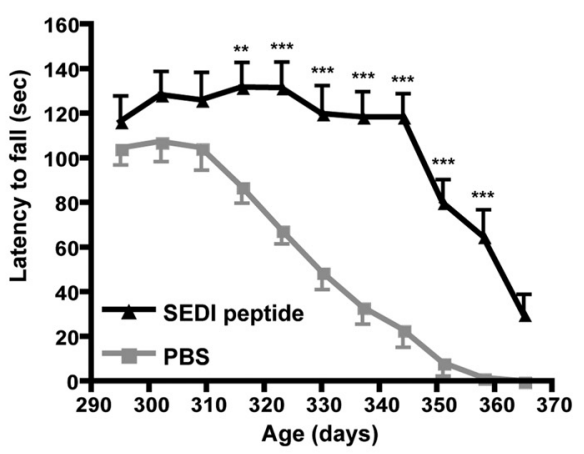

the therapeutic benefits of an active immunization approach using SEDI peptide to specifically target monomer/misfolded SOD1. SOD $1^{\mathrm{G} 37 \mathrm{R}}$ mice were immunized with SEDI peptide $(n=10)$ in CFA, using PBS in CFA (saline-CFA) as negative control $(n=10)$, from 6 weeks of age until 5 months of age. Immunization was initiated at 6 weeks of age to determine the effect of removing monomer/misfolded SOD1 on timing of disease onset. Second, immunization at an early stage would prevent potential conflicts with the neuroinflammatory contribution to the disease that is typically observed after disease onset. All treated animals were subjected to rotarod testing to analyze the effects of immunization on motor function. SOD1 ${ }^{\mathrm{G} 37 \mathrm{R}}$ mice injected with saline-CFA showed a significant decline on the rotarod task at $323 \mathrm{~d}$, and motor function continued to decline as disease progressed (Fig. 1C). In contrast, motor performance was significantly improved in SEDI peptide-immunized mice over the course of disease compared with mice injected with saline-CFA (Fig. 1C). Footprint analyses using hindlimb stride length as an index of motor function was also performed. Mice vaccinated with SEDI peptide had significant improvements in walking patterns compared with controls (Fig. $1 D, E)$. Mice were monitored for disease onset, as defined by a $20 \%$ reduction in rotarod performance. Saline-CFA-injected mice showed an onset of motor deficits at $325.7 \pm$ $3.0 \mathrm{~d}$, while the onset of motor deficits was significantly delayed to $353.2 \pm 3.7 \mathrm{~d}(p<$ 0.0001 ) in mice immunized with SEDI peptide (Fig. $1 F$ ). Clinical end stage was defined as the age when the mouse was unable to right itself within $30 \mathrm{~s}$ after being placed on its side. Animals receiving SEDI peptide immunization showed a significant increase in life span compared with those receiving saline-CFA $(392.5 \pm 3.6$ vs $352.1 \pm 1.9 \mathrm{~d}$; $p<0.0001$; Fig. $1 G$ ). Furthermore, immunization slowed disease progression as the duration of the disease in SEDI peptide was $39.3 \mathrm{~d}$ compared with $26.4 \mathrm{~d}$ for saline-CFAinjected mice.

\section{Immunization with SEDI peptide generates anti-SOD1 antibody and} Th2-biased immune response

To assess the anti-SOD1 immune responses elicited by SEDI peptide, we de-

non-Tg mice (Fig. $1 B$ ), indicating that monomer/misfolded SOD1 as well as natively folded SOD 1 is present extracellularly as well as intracellularly.

In vitro studies have shown that mutant forms of SOD1 have a higher propensity to misfold and/or to dissociate into monomers and ultimately aggregate (Valentine and Hart, 2003; Khare et al., 2004; Rakhit et al., 2004; Ray et al., 2004). We therefore evaluated termined the antibody titers from the collected serum samples of immunized mice at 6 months of age and disease end stage. At 6 months of age, low-titer antibodies to unfolded SOD1 were observed in animals immunized with SEDI peptide $(319 \pm 40 ; n=$ 10; Fig. $2 A$ ). An increase in antibody titer in immunization with SEDI peptide $(605 \pm 37 ; n=10)$ was detected at end stage (Fig. $2 A$ ). Animals receiving saline-CFA had negligible SOD1 titers at 


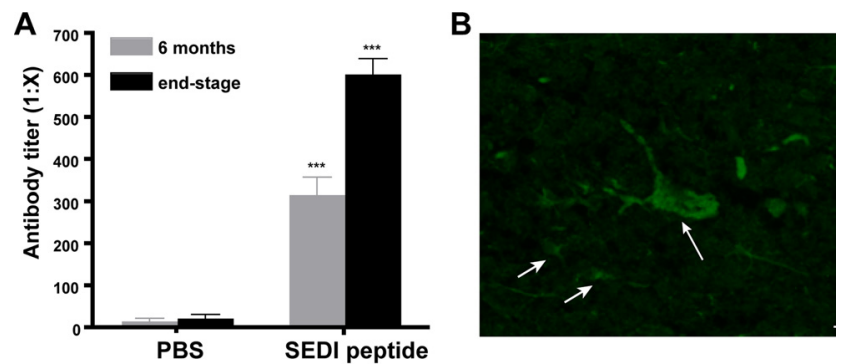

Figure 2. SEDI peptide induces a Th2-biased immune response. $A$, SOD1-specific immune responses in mice vaccinated with SEDI peptide. Serum from nonvaccinated and vaccinated mice were used to determine anti-S0D1 antibody titers. The horizontal bars represent mean titer in the respective group $(n=10)$. Values are expressed as a mean $\pm \operatorname{SEM}\left({ }^{* * *} p<0.001\right)$. $B$, Presence of IgG within a motor neuron (long arrow) of the anterior horn of the lumbar spinal cord, as well as surrounding glial cells (short arrows) of immunized animals detected with Alexa Fluor-labeled secondary antibody to mouse lgG. No labeling obtained in corresponding tissue from animals receiving saline-CFA. Scale bar, $20 \mu \mathrm{m}$.

both 6 months of age $(18 \pm 6 ; n=10)$ and end stage $(24 \pm 9 ; n=$ 10; Fig. $2 A$ ).

To further characterize the antibody response, we determined the IgG isotype, the profile of which is influenced by the Th1/Th2 balance of the immune system. Th1 response produces proinflammatory cytokines and is critical in cell-mediated immunity, while Th2 response stimulates antibody production and plays an important role in humoral immunity. The ratio of IgG1 to IgG2a antibodies produced serves as an indirect measurement of Th2and Th1-type of immune response (Finkelman et al., 1990). All mice immunized with SEDI peptide generated IgG1 antibodies and negligible levels of IgG2a (data not shown). The ratio of IgG1 to IgG2a exceeded 1 after immunization with SEDI peptide $(1.25 \pm 0.08 ; n=10)$, indicating a predominance of Th2-biased immune response. To test whether IgG was present in the spinal cord of immunized mice, frozen transverse sections from immunized and nonimmunized animals were labeled with Alexa Fluor 488 goat anti-mouse secondary antibody. Labeling of motor neurons within the ventral horn was clearly apparent in immunized animals but not in nonimmunized mice (Fig. $2 B$, arrows).

\section{Induced SOD1 antibodies recognize SOD1 in ALS transgenic mice}

To determine specificity of sera to pathologically relevant features in ALS mouse models, the immunoreactivity of actively generated sera was tested against disease tissue of untreated transgenic mice. No labeling was detected with sera from control mice (Fig. $3 A-D$ ), whereas a diffuse pattern of monomer/misfolded SOD1 with sera from SEDI peptide-immunized mice in motor neuron perikarya and neurites was detected (Fig. $3 G, H$, arrows). Interestingly, immunoreactivity to the SEDI epitope was also found in neighboring astrocytes, an observation we have made previously (Rakhit et al., 2007). Double labeling with SEDI peptide-immunized sera and GFAP, a marker for astrocytes, confirmed the presence of monomer/misfolded SOD1 in astrocytes (Fig. $3 E-G$ ). Detection of SOD1 with sera from vaccinated mice was confirmed by Western blotting (Fig. 3I). The presence of monomer/misfolded SOD1 in spinal cord lysates was detected by immunoprecipitation using sera from mice immunized with SEDI peptide and then immunoblotting with SOD1 antibody (Calbiochem) (Fig. 3I). In contrast, no SOD1 immunoreactivity was observed with sera from saline-CFA-injected mice (Fig. 3I).

\section{Immunization with SEDI peptide protects motor neurons} and reduces gliosis

To correlate the behavioral improvement with pathological outcome measures, the effect of immunization on the size and number of motor axons in L5 ventral roots was quantified. At disease onset $(320 \mathrm{~d})$, we observed a $50 \%$ reduction in the total number of motor axons in saline-CFA-injected mice (444 $\pm 23 ; n=4$; $p<0.001$; Fig. $4 A$ ) compared with non-Tg mice (951 $\pm 69 ; n=$ 4 ; Fig. $4 C$ ), whereas motor axons at the same time point in mice vaccinated with SEDI peptide (Fig. $4 B$ ) were well preserved and their number was statistically indistinguishable from non-Tg mice (853 \pm 51 in mice receiving SEDI peptide, $n=4, p>0.05$, vs $951 \pm 69$ in non-Tg mice, $n=4)$. Further analyses showed that immunization with SEDI peptide protected large motor neurons. Average counts of large motor axons $(>5 \mu \mathrm{m})$ in SEDI peptideimmunized $(711 \pm 64 ; n=4 ; p>0.05)$ animals were similar to non-Tg animals ( $813 \pm 76)$; in contrast, animals receiving salineCFA showed a $63 \%$ loss of large motor axons $(293 \pm 32$ vs $813 \pm$ $76 ; n=4 ; p<0.001$; Fig. $4 D)$.

Immunohistochemical analysis of the lumbar spinal cord also showed a substantial loss of motor neurons in CFA-treated mice as shown by $\mathrm{H} \&$ E labeling (Fig. $4 E$ ). Similarly to the preservation of motor axons, more intact and healthy motor neurons were found in mice immunized with SEDI peptide (Fig. $4 H$ ). In ALS, loss of motor neurons is accompanied by a robust inflammatory response. The cellular events consist of proliferation and activation of astrocytes and microglia at the sites of degenerating motor neurons (McGeer and McGeer, 2002; Sargsyan et al., 2005). In mutant SOD1 transgenic mice, it is well documented that activation of microglia is evident at presymptomatic stage, and astrogliosis is prominent at disease onset (Hall et al., 1998; Alexianu et al., 2001; McGeer and McGeer, 2002). To probe the effect of immunization on glial responses at disease onset (320 d), lumbar spinal cord sections from immunized mice were labeled for GFAP and Mac-2, as markers for astrogliosis and activated microglia, respectively. Consistent with previous reports, we observed intense astrogliosis and microglial activation as shown by strong immunolabeling of GFAP and Mac-2, respectively, in saline-CFA-treated mice (Fig. $4 F, G$ ). However, in mice vaccinated with SEDI peptide (Fig. $4 I, J$ ), immunoreactivity of GFAP and Mac-2 was greatly attenuated. These results indicate that immunization with SEDI peptide not only delays degeneration of motor neurons but also reduced associated astrogliosis and microglial activation.

\section{Effects of SEDI peptide immunization in SOD1 ${ }^{\text {G93A }}$ transgenic mice}

SOD $1{ }^{\mathrm{G} 93 \mathrm{~A}}$ transgenic mice overexpressing $\sim 20$ copies of the mutant gene are the most widely used animal model for testing therapeutic strategies and studying disease mechanisms in ALS. Therefore, we also assessed the effect of immunization with SEDI peptide in SOD1 ${ }^{\mathrm{G} 93 \mathrm{~A}}$ mice, an early-onset ALS mouse model (Gurney et al., 1994). Immunization was initiated at 6 weeks of age followed by a boost at 8 weeks of age and a final boost at 12 weeks of age. Both rotarod and footprint analyses were conducted weekly to evaluate motor performance. A significant decline in rotarod performance was detected in saline-CFA-injected mice at $117 \mathrm{~d}$ that progressively became worse over the course of disease (Fig. $5 A$ ). Improvements in rotarod performance (119$126 \mathrm{~d} ; p<0.05$; Fig. 5A) and stride length (130 d; $p<0.001$; Fig. $5 B$ ) were not found to be statistically significant in mice immunized with SEDI peptide. A 12 d delay in disease onset was observed in mice vaccinated with SEDI peptide $(129.6 \pm 3.8$ vs 

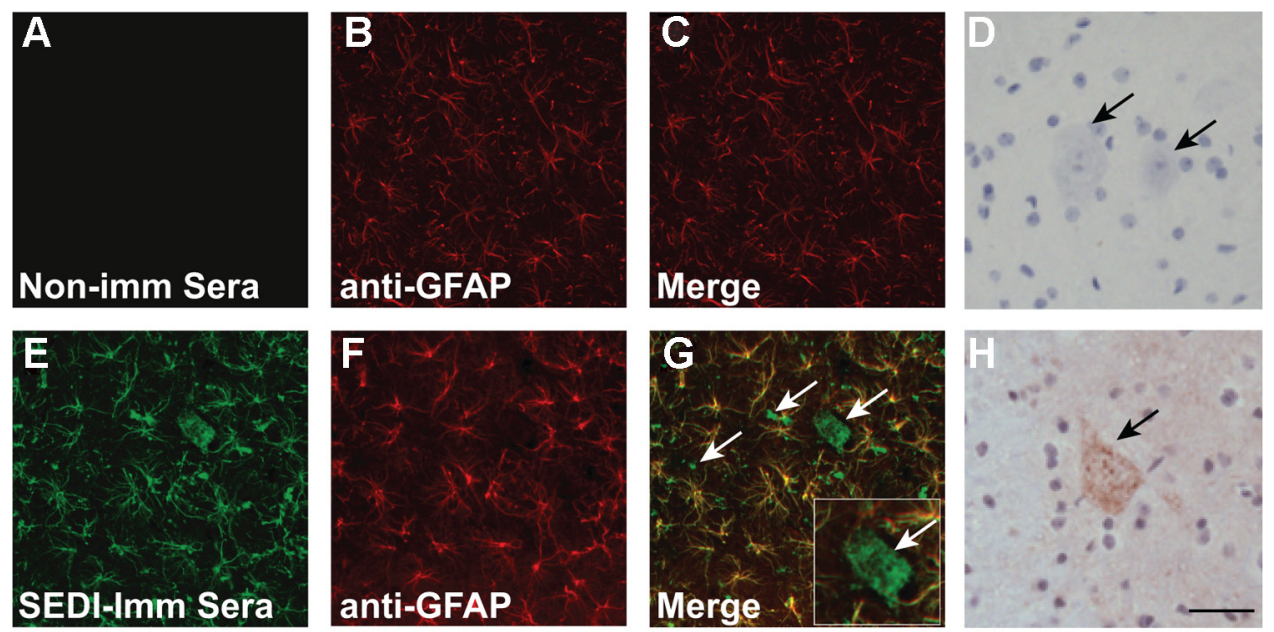

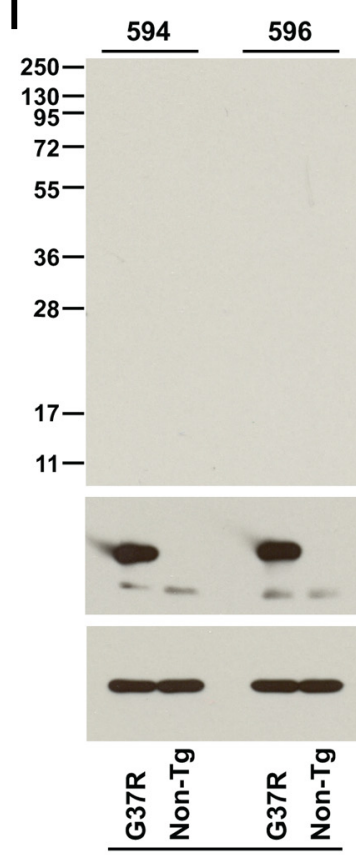

PBS

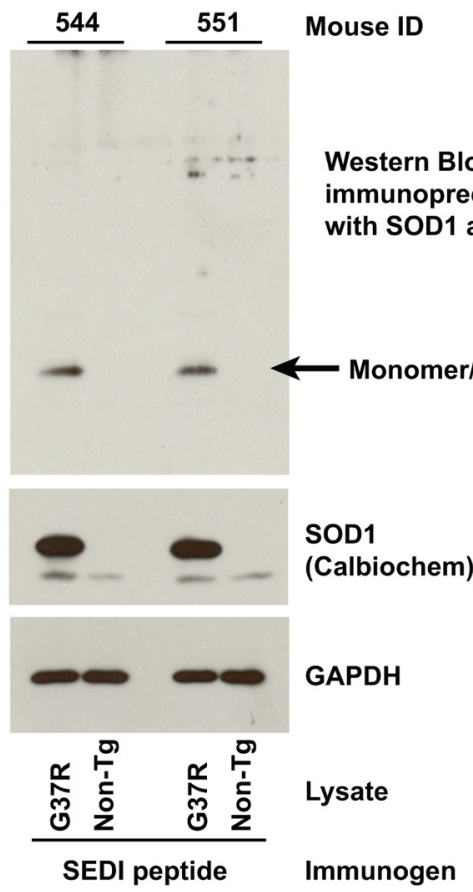

Figure 3. Sera from immunized mice recognize monomer/misfolded SOD1 in spinal cord of SOD1 ${ }^{\text {G37R }}$ mice. $\boldsymbol{A}-\boldsymbol{H}$, Sections from lumbar spinal cord of SOD1 ${ }^{\text {G37R }}$ mice were labeled with sera from saline-CFA- or SEDI peptide-immunized mice ( $\boldsymbol{A}, \boldsymbol{E}$; green) and an antibody against GFAP ( $\boldsymbol{B}, \boldsymbol{F}$; red), with the merged images shown in $\boldsymbol{C}$ and $\boldsymbol{G}$. Paraffin-embedded formalin-fixed sections colabeled with $\mathrm{H} \&$ E and the respective sera are shown in $\boldsymbol{D}$ and $\boldsymbol{H}$, with arrows indicating motor neurons. $\boldsymbol{A}-\boldsymbol{D}$, Sera from CFA-treated mice did not show any labeling. $\boldsymbol{E}$ - $\boldsymbol{H}$, Sera from mice immunized with SEDI peptide labeled monomer/misfolded SOD1 within motor neurons and astrocytes. The arrows in $\mathbf{G}$ and $\boldsymbol{H}$ indicate neuronal labeling; the inset shows higher magnification of motor neuron labeled with sera. Clearer labeling of a motor neuron is shown in $\boldsymbol{H}$. Note the reduced detection of monomer/misfolded SOD1 in the paraffin-embedded formalin-fixed section (compare $\boldsymbol{E}, \boldsymbol{H}$ ). Scale bar: $\boldsymbol{A}-\boldsymbol{C}, \boldsymbol{E}-\mathbf{G}, 15 \mu \mathrm{m} ; \boldsymbol{D}, \boldsymbol{H}, 30 \mu \mathrm{m}$. I, Detection of monomer/misfolded S0D1 with antisera from SEDI peptide-vaccinated mice was confirmed by Western blotting. Spinal cord lysates from SOD1 ${ }^{\text {G37R }}$ and non-Tg mice were subjected to immunoprecipitation using antisera from mice immunized with SEDI peptide (mouse ID 544 and 551 ) or saline-CFA (mouse ID 594 and 596). The immunoprecipitates were then probed by Western blot using SOD1 antibody (Calbiochem). Note that antisera from SEDI peptide-immunized animals immunoprecipitated monomer/misfolded SOD1 from SOD1 ${ }^{\text {G37R }}$ mice but not non-Tg mice, and that no signal was detected using antisera from saline-CFA-immunized animals. A separate immunoblot of the spinal cord lysates used as input for the immunoprecipitations shows the presence of human and/or mouse SOD1 in the SOD1 ${ }^{\text {G37R }}$ or non-Tg mice, respectively, with GAPDH used as the loading control.

$117.2 \pm 1.4 \mathrm{~d} ; p=0.0005$; Fig. $5 C)$; however, this did not translate to an significant increase in life span $(165.5 \pm 3.1$ vs $158.6 \pm 2.7 \mathrm{~d}$; $p=0.0528$; Fig. 5D).

\section{SEDI peptide immunization reduces monomer/misfolded} and oligomeric SOD1

We further explored the molecular mechanisms mediating the therapeutic effects of SOD1 immunization. Studies have shown that mutant SOD1 forms aggregates in vitro (Rakhit et al., 2002; Rodriguez et al., 2002; Stathopulos et al., 2003), and highmolecular-weight SOD1 species are apparent on immunoblots of spinal cord lysates from transgenic animals (Johnston et al., 2000; Wang et al., 2002; Furukawa et al., 2006). Therefore, the improvement of motor function and mortality may stem from the reduction of oligomeric/aggregated SOD1 species. To address this, we measured the presence of high-molecular-weight SOD1 oligomers by immunoblot analyses. In mice receiving saline-CFA, SOD1 oligomers were found in lumbar spinal cord extracts at $320 \mathrm{~d}$ (Fig. $6 \mathrm{~A})$. In contrast, immunization with SEDI peptide reduced the accumulation of SOD1 oligomers by $92.6 \pm 24.6 \%(n=4$; $p<0.01$ ) compared with the level in control mice (Fig. 6A). Using SEDI antibody to immunoprecipitate monomer/mis- 
folded SOD1, we found a $35.2 \pm 6.5 \%$ $(n=4 ; p<0.05)$ decrease in monomer/ misfolded SOD1 after immunization with SEDI peptide compared with control (Fig. 6B).

\section{Discussion}

Although previous immunization studies have targeted pathological forms of SOD1 in mutant SOD1 transgenic mice, the precise epitopes targeted are unknown (Urushitani et al., 2007; Gros-Louis et al., 2010; Takeuchi et al., 2010). Here, we have used SEDI peptide in an immunization strategy to specifically target monomer/misfolded SOD1 species in which the dimer interface is exposed (Rakhit et al., 2007). The therapeutic benefits of this strategy included a delay in disease onset, extension of disease progression, and prolongation of survival by up to $40 \mathrm{~d}$. Importantly the SEDI peptide antigen directed a Th2-biased immune response, and this led to a reduction in monomer/misfolded SOD1, the intended target of the immunization strategy. This provides the first evidence for the neurotoxicity of monomeric/misfolded SOD1 in vivo and validates these species as legitimate therapeutic targets for the treatment of SOD1 associated ALS.

There are $>130$ different mutations found in the SOD1 gene in ALS (http:// alsod.iop.kcl.ac.uk). The mode of toxicity of the different mutations is thought to be through induction of misfolding and/or aggregation of the mutant SOD1 protein as an upstream event leading to a multipathway neurodegenerative cascade that causes motor neuron degeneration, as well as the accompanying pathological changes, such as gliosis, that are typically associated with ALS. Mutations in SOD1 have been shown to promote SOD1 misfolding/aggregation through at least three distinct mechanisms: reducing the net charge, impairing metal binding, or destabilizing the native structure (DiDonato et al., 2003; Khare et al., 2004; Leinweber et al., 2004; Stathopulos et al., 2006; Shaw and Valentine, 2007). In vitro aggregation assays of SOD1 have identified a multistep process in which the SOD1 dimer is destabilized, forming a monomeric species before the formation of aggregates (Rakhit et al., 2002, 2004; Khare et al., 2004). Evidence for this mechanism in vivo was shown using the SEDI antibody, which detected monomeric/misfolded SOD1 in three lines of mutant SOD1 transgenic mice (G37R, G85R, and G93A) and in five different human fALS1 cases (A4T, A4V, V14M, $\Delta \mathrm{G} 27 / \mathrm{P} 28$, and I113T) (Rakhit et al., 2007; Liu et al., 2009), indicating that there is a common misfolding pathway among the different SOD1 mutant proteins in vivo. In this regard, we used SEDI peptide as antigen in an immunization strategy aimed at specifically targeting this common misfolded species, monomer/misfolded SOD1, in mutant SOD1 transgenic mice. Induced antibody titers
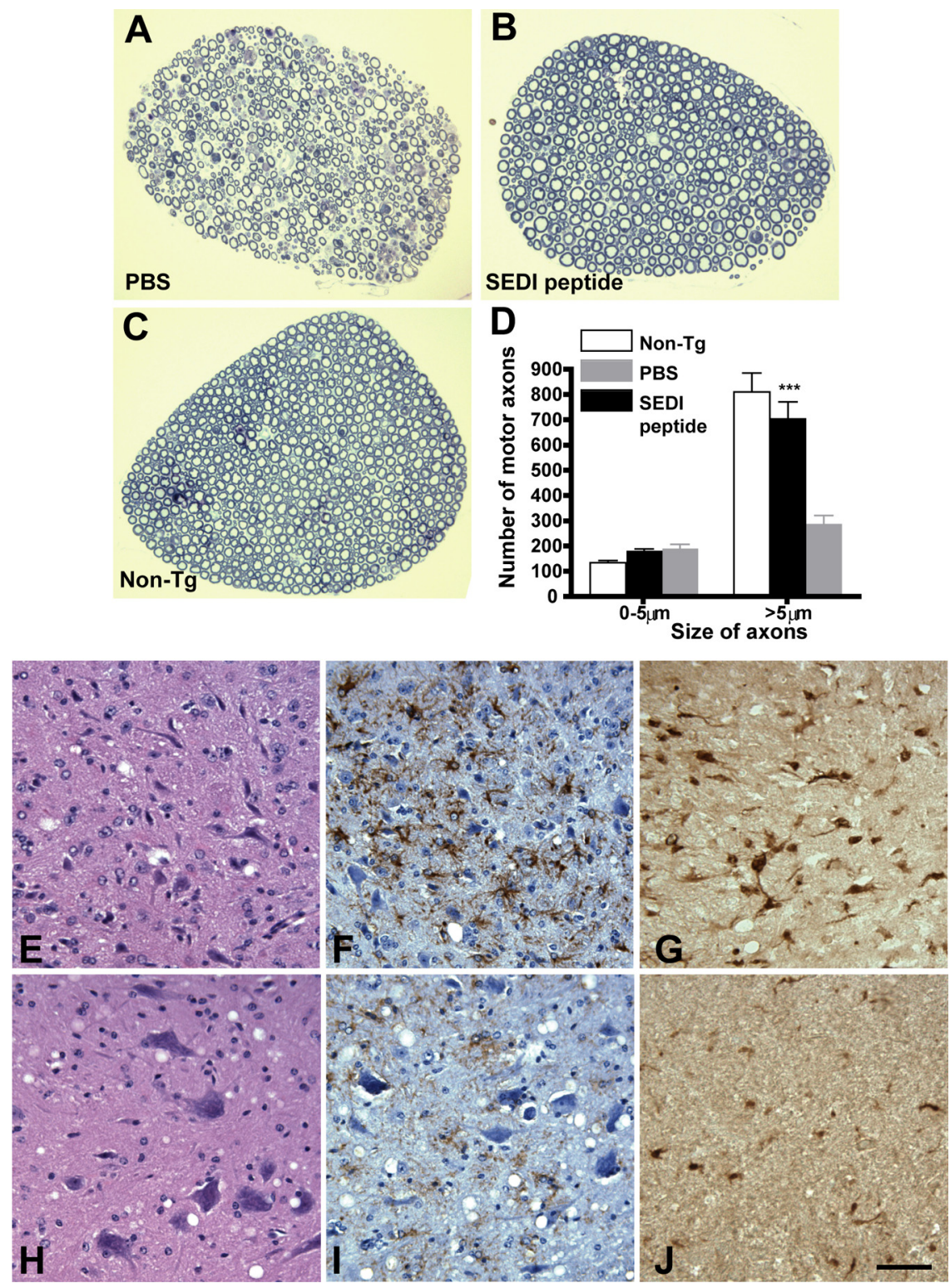

Figure 4. SOD1 immunization improves motor neuron survival and reduces gliosis in S0D1 ${ }^{\text {G37R }}$ mice. $\boldsymbol{A}-\boldsymbol{C}$, Representative light micrographs of toluidine blue-stained transverse $L 5$ ventral root section at $320 \mathrm{~d}$. SEDI immunization conferred a protective effect SEDI peptide significantly decreased motor neuron loss (compare $\boldsymbol{E}, \boldsymbol{H}$ ), astrogliosis (compare $\boldsymbol{F}, \boldsymbol{I}$ ), and activated microglia (compare $\mathbf{G}, \boldsymbol{J}$ ) compared with nonvaccinated animals, as evidenced by labeling lumbar spinal cord of 320 -d-old immunized mice with H\&E and antibodies against GFAP and Mac-2, respectively.

were detectable at 6 months of age in the immunized SOD1 ${ }^{\text {G37R }}$ mice and increased approximately twofold by $\sim 10$ months of age. Sera from mice immunized with SEDI peptide labeled SOD1 aggregates in diseased spinal motor neurons and gave no labeling of control nontransgenic mouse spinal cord. Furthermore, sera from the SEDI-immunized animals immunoprecipitated monomeric/misfolded SOD1 from diseased tissue, confirming the selectivity of the induced antisera for pathological SOD1. Importantly, immunization with SEDI peptide directed a Th2-biased immune response, which promotes antibody production and suppresses the Th1-type proinflammatory response. Of note, it was a Th1 immune response that led to meningoencephalitis in a subset of patients receiving immunizations of $A \beta$ peptide in the AN1792 clinical trial for Alzheimer's disease (for review, see 
A

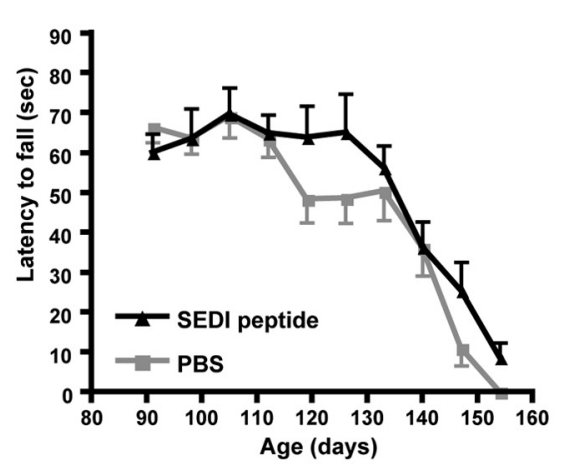

C

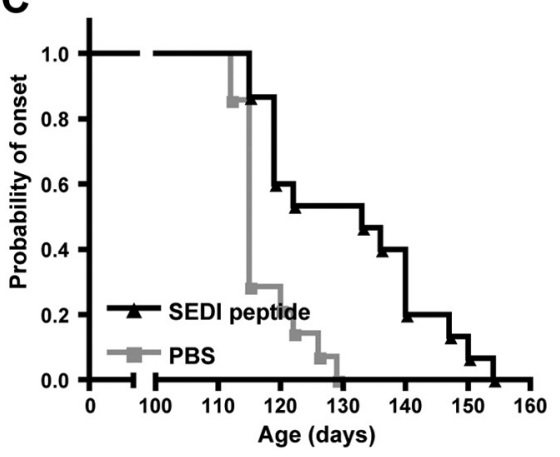

B

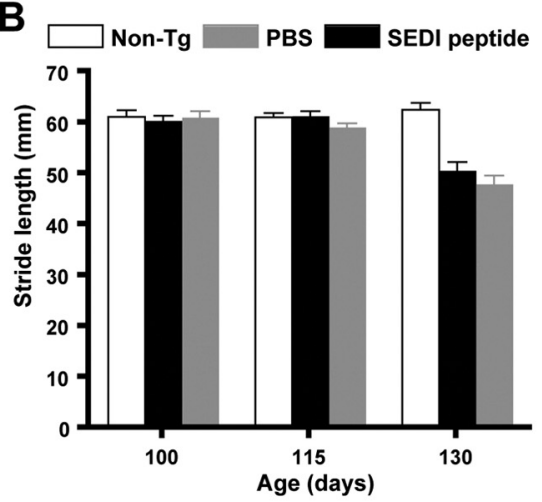

D

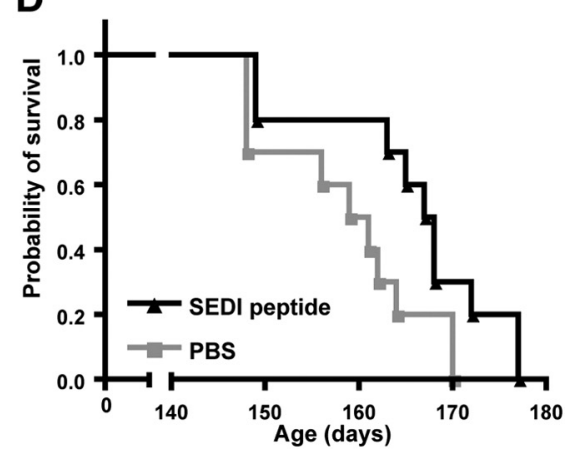

Figure 5. SEDI immunization confers modest protection on disease onset in SOD1 ${ }^{693 A}$ mouse model. Rotarod task $(\boldsymbol{A})$ and footprint analysis $(\boldsymbol{B})$ were performed to evaluate motor impairment in immunized SOD1 ${ }^{\mathrm{G} 93 \mathrm{~A}}$ mice. Immunization with SEDI peptide (gray; $n=10$ ) had no significant effect on rotarod and footprint analyses. Values are expressed as mean \pm SEM $(\boldsymbol{A}, \boldsymbol{B}$; $\left.{ }^{*} p<0.05,{ }^{* * *} p<0.001\right)$. Age of disease onset ( $\left(\right.$ ) and survival (D) in SOD $1{ }^{693 A}$ CFA-treated mice (black; $n=9$ ) and SEDI peptide (gray; $n=10$ )-immunized mice. Immunization with SEDI peptide significantly delayed disease onset but did not significantly prolong survival.

A

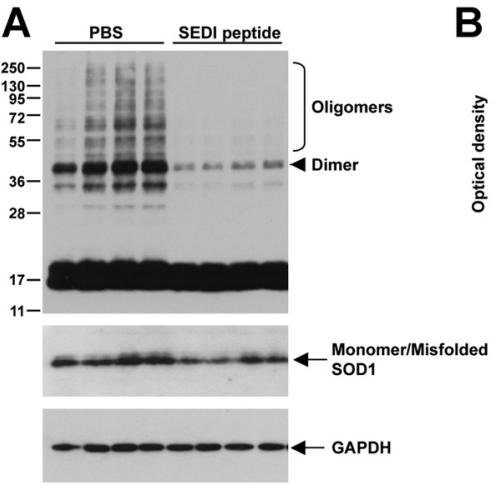

Figure 6. SEDI peptide immunization reduces monomer/misfolded and oligomeric SOD1. $A$, Western blot analysis of the levels of SOD1 oligomers and misfolded SOD1 in lumbar spinal cord of immunized animals at $320 \mathrm{~d}$. Compared with CFA-treated mice, high-molecular-weight oligomers were greatly reduced in SEDI peptide-immunized mice. The level of monomer/misfolded SOD1 was also reduced in mice vaccinated with SEDI peptide. GAPDH was used as a loading control. B, Quantification of SOD1 oligomers and monomer/misfolded SOD1 in immunized mice. Values are expressed as mean \pm SEM $\left({ }^{*} p<0.05,{ }^{* *} p<0.01\right.$, $\left.{ }^{* * *} p<0.001 ; n=5\right)$.

Menéndez-González et al., 2011). Interestingly, Urushitani and colleagues (Takeuchi et al., 2010) have shown that immunization with WT-apo SOD1 induces a higher Th2/Th1 milieu than G93A-apo SOD1 vaccination, indicating the importance of antigen selection in obtaining the desired outcomes. In this regard, here we have shown that SEDI peptide gave a favorable Th2biased immune response.
The therapeutic targeting of monomer/misfolded SOD1 delayed disease onset by an average of $25 \mathrm{~d}$ in SOD $1^{\text {G37R }}$ transgenic mice, further implying that these species contribute to the earliest stages of disease. SEDI peptide immunization also delayed disease onset by $12 \mathrm{~d}$ in the SOD1 ${ }^{\text {G93A }}$ transgenic mice, which was not observed when recombinant mutant SOD1 protein was used as immunogen (Urushitani et al., 2007), perhaps suggesting that SEDI peptide is a more efficacious immunogen, at least in this system. Immunization with SEDI peptide not only protected motor neurons, the primary target of SOD1 toxicity, but also reduced neuroinflammatory responses including astrogliosis and microgliosis, implying that these events are downstream from the generation of monomer/misfolded SOD1. Non-cell-autonomous toxicity is well documented in transgenic SOD1 mice (Clement et al., 2003; Beers et al., 2006; Boillée et al., 2006). Suppression of inflammatory responses has had various effects primarily on disease duration in mutant SOD 1 transgenic mice (Kriz et al., 2002; Zhu et al., 2002; Klivenyi et al., 2004), and by extension our results show that amelioration of astrogliosis and microgliosis also correlates with a lengthening of disease duration. The preferential Th2 immune response in our immunization study may also have contributed to the reduced inflammatory responses. Significantly, monomer/ misfolded SOD1 was detected in the CSF of mutant SOD1 transgenic mice, and based on earlier studies describing the neurotoxicity of extracellular SOD1 either directly or through activation of glial cells (Urushitani et al., 2007), a potential mechanism of the immunization strategy is to clear misfolded SOD1 from the extracellular milieu. In this regard, although a number of theories have been promulgated for the mechanistic action of immunization for the treatment various neurodegenerative diseases (Masliah et al., 2005; Asuni et al., 2007; Urushitani et al., 2007; Takeuchi et al., 2010), as with these earlier studies, we were also unable to ascertain which pools of monomer/misfolded SOD1 our immunization strategy targeted, extracellular or intracellular. It is possible that there is Fc receptor-mediated phagocytosis of SOD1/antibody complexes by microglia, as shown in animal models of Alzheimer's disease (Bard et al., 2000). As mentioned, the presence of misfolded SOD1 in the CSF would be consistent with this mode of action. The clearance of intracellular SOD1 aggregates might involve the entrance of anti-SOD1 antibodies into motor neurons. Supporting evidence for this mechanism came from reports that IgG can be taken up by motor neuron terminals and transported retrogradely to the cell body (Fabian and Petroff, 1987; Mohamed et al., 2002). Indeed, IgG reactivity was found in motor neurons in the spinal cord of ALS cases (Engelhardt and Appel, 1990), and we detected IgG within motor neuron perikarya of immunized mice. Internalized antibodies may exert an inhibitory effect on SOD1 aggregation and/or trigger the endosomal/lysosomal pathway, as has been described previously (Masliah et al., 2005; Asuni et al., 2007). 
Importantly, we ascertained using immunoprecipitation studies from spinal cord that there was an overall reduction in the amount of monomer/misfolded SOD1 in SEDI peptideimmunized animals relative to controls, the intended target of the immunization strategy. We also found a reduction in highermolecular-weight oligomeric SOD1 species. It appears from these results that monomer/misfolded SOD1 is a precursor required to form the higher-molecular-weight oligomeric SOD1 species. We were unable to ascertain from our results whether it is the monomer/misfolded SOD1 itself that is the toxic entity, or whether it is the oligomeric species that are toxic, and this is part of an ongoing study. SEDI peptide immunization prolonged survival of SOD $1^{\text {G37R }}$ transgenic mice by an average of $40 \mathrm{~d}$, and although this may seem modest for an animal model exhibiting an average life span of $325 \mathrm{~d}$, it should be borne in mind that only small amounts of antibody cross the blood-brain barrier $[\sim 0.1 \%$ of serum levels (Bard et al., 2000)], which is likely to be insufficient to counter the fivefold overexpression of the mutant SOD1 protein in the SOD ${ }^{\text {G37R }}$ mice. This is supported by the fact that, although immunization modestly delayed disease onset in the SOD1 ${ }^{\text {G93A }}$ transgenic mouse in which there is a $\sim 20$-fold overexpression of mutant SOD1, there was no prolongation of survival. Furthermore, there may be additional pathological SOD1 species, not targeted by our immunization strategy, that also contribute to the pathogenic process (Urushitani et al., 2007; Bosco et al., 2010; Gros-Louis et al., 2010; Takeuchi et al., 2010; Pokrishevsky et al., 2012). In this regard, there is evidence that C4F6 antibody labels "mal-folded" forms of SOD1 in transfected cell lines (Prudencio and Borchelt, 2011), and a series of peptide antibodies have been produced that detect chemically denatured non-native forms of SOD1 (Forsberg et al., 2010). Both sets of antibodies have been reported to label diffuse and/or small punctate-like SOD1 inclusions of $0.5-3 \mu \mathrm{m}$ in sporadic ALS (Bosco et al., 2010; Forsberg et al., 2010), although recently it was reported that labeling is also obtained using C4F6 antibody in control tissues (Brotherton et al., 2012). In contrast, SEDI antibody appears specific for fALS1 mutant SOD1 and has been verified as labeling all SOD1 mutants tested to date in both fALS1 cases (A4V, A4T, I113T, G27/P28, V14M), labeling the characteristic hyaline conglomerate inclusions (Hays, 2006; Hays et al., 2006), and in mutant SOD1 transgenic mice (G37R, G93A, G85R) (Liu et al., 2009), and this strengthens the argument for targeting monomer/misfolded SOD1 therapeutically in fALS1 cases.

The immunization strategy that we propose here is aimed at removing monomer/misfolded SOD1 as it is produced to ameliorate the disease. Another strategy currently undergoing clinical trials is antisense oligonucleotide therapy. In this approach, expression of the mutant gene is downregulated by the targeted antisense oligonucleotide (AON), reducing the total amount of mutant SOD1 protein generated (Smith et al., 2006). Although more invasive than immunization, this approach holds great promise and may prove to be most effective when administered before disease onset. However, it is also likely that some mutant SOD1 protein will continue to be generated, escaping the effect of the AON and form toxic monomer/misfolded SOD1. In this case, AON could be combined with an immunization strategy to remove monomer/misfolded SOD1 as a "second hit" to effectively treat the disease. Importantly, there is increasing evidence that propagation of neurodegenerative diseases, including ALS, are propagated by the cell-cell transmissibility of pathological misfolded proteins (Grad et al., 2011; Prudencio and Borchelt, 2011; de Calignon et al., 2012). We have shown here that monomer/ misfolded SOD1 is present within the extracellular milieu, and as such, SEDI peptide immunization could be important to halting or slowing the progression of the disease by preventing cell-cell transmissibility of monomer/misfolded of SOD1.

In summary, our findings show that an immunotherapeutic approach specifically targeting monomer/misfolded SOD1 ameliorates disease phenotype and pathology in mutant SOD1 transgenic mice. These findings not only indicate the pathogenic nature of these species but also identify monomer/misfolded SOD1 as a valid therapeutic target for the treatment of ALS.

\section{References}

Alexianu ME, Kozovska M, Appel SH (2001) Immune reactivity in a mouse model of familial ALS correlates with disease progression. Neurology 57:1282-1289.

Asuni AA, Boutajangout A, Quartermain D, Sigurdsson EM (2007) Immunotherapy targeting pathological tau conformers in a tangle mouse model reduces brain pathology with associated functional improvements. J Neurosci 27:9115-9129.

Azzouz M, Ralph GS, Storkebaum E, Walmsley LE, Mitrophanous KA, Kingsman SM, Carmeliet P, Mazarakis ND (2004) VEGF delivery with retrogradely transported lentivector prolongs survival in a mouse ALS model. Nature 429:413-417.

Bard F, Cannon C, Barbour R, Burke RL, Games D, Grajeda H, Guido T, Hu K, Huang J, Johnson-Wood K, Khan K, Kholodenko D, Lee M, Lieberburg I, Motter R, Nguyen M, Soriano F, Vasquez N, Weiss K, Welch B, et al. (2000) Peripherally administered antibodies against amyloid betapeptide enter the central nervous system and reduce pathology in a mouse model of Alzheimer disease. Nat Med 6:916-919.

Beers DR, Henkel JS, Xiao Q, Zhao W, Wang J, Yen AA, Siklos L, McKercher SR, Appel SH (2006) Wild-type microglia extend survival in PU.1 knockout mice with familial amyotrophic lateral sclerosis. Proc Natl Acad Sci U S A 103:16021-16026.

Boillée S, Yamanaka K, Lobsiger CS, Copeland NG, Jenkins NA, Kassiotis G, Kollias G, Cleveland DW (2006) Onset and progression in inherited ALS determined by motor neurons and microglia. Science 312:1389-1392.

Bosco DA, Morfini G, Karabacak NM, Song Y, Gros-Louis F, Pasinelli P, Goolsby H, Fontaine BA, Lemay N, McKenna-Yasek D, Frosch MP, Agar JN, Julien JP, Brady ST, Brown RH Jr (2010) Wild-type and mutant SOD1 share an aberrant conformation and a common pathogenic pathway in ALS. Nat Neurosci 13:1396-1403.

Brotherton TE, Li Y, Cooper D, Gearing M, Julien JP, Rothstein JD, Boylan K, Glass JD (2012) Localization of a toxic form of superoxide dismutase 1 protein to pathologically affected tissues in familial ALS. Proc Natl Acad Sci U S A 109:5505-5510.

Bruijn LI, Miller TM, Cleveland DW (2004) Unraveling the mechanisms involved in motor neuron degeneration in ALS. Annu Rev Neurosci 27:723-749.

Clement AM, Nguyen MD, Roberts EA, Garcia ML, Boillée S, Rule M, McMahon AP, Doucette W, Siwek D, Ferrante RJ, Brown RH Jr, Julien JP, Goldstein LS, Cleveland DW (2003) Wild-type nonneuronal cells extend survival of SOD1 mutant motor neurons in ALS mice. Science 302:113-117.

de Calignon A, Polydoro M, Suárez-Calvet M, William C, Adamowicz DH, Kopeikina KJ, Pitstick R, Sahara N, Ashe KH, Carlson GA, Spires-Jones TL, Hyman BT (2012) Propagation of tau pathology in a model of early Alzheimer's disease. Neuron 73:685-697.

DiDonato M, Craig L, Huff ME, Thayer MM, Cardoso RM, Kassmann CJ, Lo TP, Bruns CK, Powers ET, Kelly JW, Getzoff ED, Tainer JA (2003) ALS mutants of human superoxide dismutase form fibrous aggregates via framework destabilization. J Mol Biol 332:601-615.

Engelhardt JI, Appel SH (1990) IgG reactivity in the spinal cord and motor cortex in amyotrophic lateral sclerosis. Arch Neurol 47:1210-1216.

Ezzi SA, Urushitani M, Julien JP (2007) Wild-type superoxide dismutase acquires binding and toxic properties of ALS-linked mutant forms through oxidation. J Neurochem 102:170-178.

Fabian RH, Petroff G (1987) Intraneuronal IgG in the central nervous system: uptake by retrograde axonal transport. Neurology 37:1780-1784.

Finkelman FD, Holmes J, Katona IM, Urban JF Jr, Beckmann MP, Park LS, Schooley KA, Coffman RL, Mosmann TR, Paul WE (1990) Lymphokine 
control of in vivo immunoglobulin isotype selection. Annu Rev Immunol 8:303-333.

Forsberg K, Jonsson PA, Andersen PM, Bergemalm D, Graffmo KS, Hultdin M, Jacobsson J, Rosquist R, Marklund SL, Brännström T (2010) Novel antibodies reveal inclusions containing non-native SOD1 in sporadic ALS patients. PLoS One 5:e11552.

Furukawa Y, Fu R, Deng HX, Siddique T, O’Halloran TV (2006) Disulfide cross-linked protein represents a significant fraction of ALS-associated $\mathrm{Cu}, \mathrm{Zn}$-superoxide dismutase aggregates in spinal cords of model mice. Proc Natl Acad Sci U S A 103:7148-7153.

Grad LI, Guest WC, Yanai A, Pokrishevsky E, O’Neill MA, Gibbs E, Semenchenko V, Yousefi M, Wishart DS, Plotkin SS, Cashman NR (2011) Intermolecular transmission of superoxide dismutase 1 misfolding in living cells. Proc Natl Acad Sci U S A 108:16398-16403.

Gros-Louis F, Soucy G, Larivière R, Julien JP (2010) Intracerebroventricular infusion of monoclonal antibody or its derived Fab fragment against misfolded forms of SOD1 mutant delays mortality in a mouse model of ALS. J Neurochem 113:1188-1199.

Gruzman A, Wood WL, Alpert E, Prasad MD, Miller RG, Rothstein JD, Bowser R, Hamilton R, Wood TD, Cleveland DW, Lingappa VR, Liu J (2007) Common molecular signature in SOD1 for both sporadic and familial amyotrophic lateral sclerosis. Proc Natl Acad Sci U S A 104:12524-12529.

Gurney ME, Pu H, Chiu AY, Dal Canto MC, Polchow CY, Alexander DD, Caliendo J, Hentati A, Kwon YW, Deng HX (1994) Motor neuron degeneration in mice that express a human $\mathrm{Cu}, \mathrm{Zn}$-superoxide dismutase mutation. Science 264:1772-1775.

Hall ED, Oostveen JA, Gurney ME (1998) Relationship of microglial and astrocytic activation to disease onset and progression in a transgenic model of familial ALS. Glia 23:249-256.

Hays AP (2006) Pathology of amyotrophic lateral sclerosis. In: Amyotrophic lateral sclerosis (Mitsumoto H, Przedborski S, Gordon PH, eds), pp 43-80. New York: Taylor and Francis Group.

Hays AP, Naini A, He CZ, Mitsumoto H, Rowland LP (2006) Sporadic amyotrophic lateral sclerosis and breast cancer: hyaline conglomerate inclusions lead to identification of SOD1 mutation. J Neurol Sci 242:67-69.

Johnston JA, Dalton MJ, Gurney ME, Kopito RR (2000) Formation of high molecular weight complexes of mutant $\mathrm{Cu}, \mathrm{Zn}$-superoxide dismutase in a mouse model for familial amyotrophic lateral sclerosis. Proc Natl Acad Sci U S A 97:12571-12576.

Khare SD, Caplow M, Dokholyan NV (2004) The rate and equilibrium constants for a multistep reaction sequence for the aggregation of superoxide dismutase in amyotrophic lateral sclerosis. Proc Natl Acad Sci U S A 101:15094-15099.

Klivenyi P, Kiaei M, Gardian G, Calingasan NY, Beal MF (2004) Additive neuroprotective effects of creatine and cyclooxygenase 2 inhibitors in a transgenic mouse model of amyotrophic lateral sclerosis. J Neurochem $88: 576-582$

Kriz J, Nguyen MD, Julien JP (2002) Minocycline slows disease progression in a mouse model of amyotrophic lateral sclerosis. Neurobiol Dis 10:268-278.

Leinweber B, Barofsky E, Barofsky DF, Ermilov V, Nylin K, Beckman JS (2004) Aggregation of ALS mutant superoxide dismutase expressed in Escherichia coli. Free Radic Biol Med 36:911-918.

Liu HN, Sanelli T, Horne P, Pioro EP, Strong MJ, Rogaeva E, Bilbao J, Zinman L, Robertson J (2009) Lack of evidence of monomer/misfolded superoxide dismutase-1 in sporadic amyotrophic lateral sclerosis. Ann Neurol 66:75-80.

Masliah E, Rockenstein E, Adame A, Alford M, Crews L, Hashimoto M, Seubert P, Lee M, Goldstein J, Chilcote T, Games D, Schenk D (2005) Effects of alpha-synuclein immunization in a mouse model of Parkinson's disease. Neuron 46:857-868.

McGeer PL, McGeer EG (2002) Inflammatory processes in amyotrophic lateral sclerosis. Muscle Nerve 26:459-470.

Menéndez-González M, Pérez-Piñera P, Martínez-Rivera M, Muñiz AL, Vega JA (2011) Immunotherapy for Alzheimer's disease: rational basis in ongoing clinical trials. Curr Pharm Des 17:508-520.

Mohamed HA, Mosier DR, Zou LL, Siklós L, Alexianu ME, Engelhardt JI, Beers DR, Le WD, Appel SH (2002) Immunoglobulin Fc gamma receptor promotes immunoglobulin uptake, immunoglobulin-mediated calcium increase, and neurotransmitter release in motor neurons. J Neurosci Res 69:110-116.

Pokrishevsky E, Grad LI, Yousefi M, Wang J, Mackenzie IR, Cashman NR
(2012) Aberrant localization of FUS and TDP43 is associated with misfolding of SOD1 in amyotrophic lateral sclerosis. PLoS One 7:e35050.

Prudencio M, Borchelt DR (2011) Superoxide dismutase 1 encoding mutations linked to ALS adopts a spectrum of misfolded states. Mol Neurodegener 6:77.

Rakhit R, Cunningham P, Furtos-Matei A, Dahan S, Qi XF, Crow JP, Cashman NR, Kondejewski LH, Chakrabartty A (2002) Oxidation-induced misfolding and aggregation of superoxide dismutase and its implications for amyotrophic lateral sclerosis. J Biol Chem 277:47551-47556.

Rakhit R, Crow JP, Lepock JR, Kondejewski LH, Cashman NR, Chakrabartty A (2004) Monomeric Cu, $\mathrm{Zn}$-superoxide dismutase is a common misfolding intermediate in the oxidation models of sporadic and familial amyotrophic lateral sclerosis. J Biol Chem 279:15499-15504.

Rakhit R, Robertson J, Vande Velde C, Horne P, Ruth DM, Griffin J, Cleveland DW, Cashman NR, Chakrabartty A (2007) An immunological epitope selective for pathological monomer-misfolded SOD1 in ALS. Nat Med 13:754-759.

Ray SS, Nowak RJ, Strokovich K, Brown RH Jr, Walz T, Lansbury PT Jr (2004) An intersubunit disulfide bond prevents in vitro aggregation of a superoxide dismutase-1 mutant linked to familial amytrophic lateral sclerosis. Biochemistry 43:4899-4905.

Rodriguez JA, Valentine JS, Eggers DK, Roe JA, Tiwari A, Brown RH Jr, Hayward LJ (2002) Familial amyotrophic lateral sclerosis-associated mutations decrease the thermal stability of distinctly metallated species of human copper/ zinc superoxide dismutase. J Biol Chem 277:15932-15937.

Rosen DR, Siddique T, Patterson D, Figlewicz DA, Sapp P, Hentati A, Donaldson D, Goto J, O’Regan JP, Deng HX (1993) Mutations in Cu/Zn superoxide dismutase gene are associated with familial amyotrophic lateral sclerosis. Nature 362:59-62.

Sargsyan SA, Monk PN, Shaw PJ (2005) Microglia as potential contributors to motor neuron injury in amyotrophic lateral sclerosis. Glia 51:241-253.

Shaw BF, Valentine JS (2007) How do ALS-associated mutations in superoxide dismutase 1 promote aggregation of the protein? Trends Biochem Sci 32:78-85.

Smith RA, Miller TM, Yamanaka K, Monia BP, Condon TP, Hung G, Lobsiger CS, Ward CM, McAlonis-Downes M, Wei H, Wancewicz EV, Bennett CF, Cleveland DW (2006) Antisense oligonucleotide therapy for neurodegenerative disease. J Clin Invest 116:2290-2296.

Stathopulos PB, Rumfeldt JA, Scholz GA, Irani RA, Frey HE, Hallewell RA, Lepock JR, Meiering EM (2003) Cu/Zn superoxide dismutase mutants associated with amyotrophic lateral sclerosis show enhanced formation of aggregates in vitro. Proc Natl Acad Sci U S A 100:7021-7026.

Stathopulos PB, Rumfeldt JA, Karbassi F, Siddall CA, Lepock JR, Meiering EM (2006) Calorimetric analysis of thermodynamic stability and aggregation for apo and holo amyotrophic lateral sclerosis-associated Gly-93 mutants of superoxide dismutase. J Biol Chem 281:6184-6193.

Takeuchi S, Fujiwara N, Ido A, Oono M, Takeuchi Y, Tateno M, Suzuki K, Takahashi R, Tooyama I, Taniguchi N, Julien JP, Urushitani M (2010) Induction of protective immunity by vaccination with wild-type apo superoxide dismutase 1 in mutant SOD1 transgenic mice. J Neuropathol Exp Neurol 69:1044-1056.

Turner BJ, Atkin JD, Farg MA, Zang DW, Rembach A, Lopes EC, Patch JD, Hill AF, Cheema SS (2005) Impaired extracellular secretion of mutant superoxide dismutase 1 associates with neurotoxicity in familial amyotrophic lateral sclerosis. J Neurosci 25:108-117.

Urushitani M, Ezzi SA, Julien JP (2007) Therapeutic effects of immunization with mutant superoxide dismutase in mice models of amyotrophic lateral sclerosis. Proc Natl Acad Sci U S A 104:2495-2500.

Valentine JS, Hart PJ (2003) Misfolded CuZnSOD and amyotrophic lateral sclerosis. Proc Natl Acad Sci U S A 100:3617-3622.

Wang J, Xu G, Borchelt DR (2002) High molecular weight complexes of mutant superoxide dismutase 1: age-dependent and tissue-specific accumulation. Neurobiol Dis 9:139-148.

Wong PC, Pardo CA, Borchelt DR, Lee MK, Copeland NG, Jenkins NA, Sisodia SS, Cleveland DW, Price DL (1995) An adverse property of a familial ALSlinked SOD1 mutation causes motor neuron disease characterized by vacuolar degeneration of mitochondria. Neuron 14:1105-1116.

Zhu S, Stavrovskaya IG, Drozda M, Kim BY, Ona V, Li M, Sarang S, Liu AS, Hartley DM, Wu DC, Gullans S, Ferrante RJ, Przedborski S, Kristal BS, Friedlander RM (2002) Minocycline inhibits cytochrome $c$ release and delays progression of amyotrophic lateral sclerosis in mice. Nature 417:74-78. 